\title{
CARACTERIZAÇÃo dA FUSÃo PERITÉTICA DO CLORETO DE SÓDIO DI-HIDRATADO POR CURVAS DE AQUECIMENTO
}

\section{Edward de Souza}

Departamento de Química, Instituto de Ciências Exatas, Universidade Federal de Minas Gerais, Av. Antonio Carlos, 6627, 31270-901 Belo Horizonte - MG, Brasil

Lucilia Aparecida Ramos de Oliveira* e Francisco Javier Rios

Serviço de Tecnologia Mineral, Centro de Desenvolvimento da Tecnologia Nuclear, Comissão Nacional de Energia Nuclear, CP 941, 30123-970 Belo Horizonte - MG, Brasil

Recebido em 5/6/08; aceito em 24/4/09; publicado na web em 22/9/09

\begin{abstract}
SODIUM CHLORIDE DIHYDRATE PERITETIC MELTING CHARACTERIZATION THROUGH HEATING CURVES. Binary mixture phase diagrams are normally obtained from thermal analysis involving freezing point curves. However, that approach is not always reliable and easy to follow to all kinds of mixtures in any proportion. In fact, even for a simple system, such as $\mathrm{NaCl}-\mathrm{H}_{2} \mathrm{O}$, this freezing methodology gives mixed results when one starts from a solid-solution system, due mostly to the formation of the $\mathrm{NaCl} .2 \mathrm{H}_{2} \mathrm{O}$, which has an incongruent melting point, and the dependence of its solubility with the temperature. In this work we report a trustworthy, simple and cheap method involving heating curves to drawn the $\mathrm{NaCl}-\mathrm{H}_{2} \mathrm{O}$ phase diagram.
\end{abstract}

Keywords: phase diagram; incongruent melting; sodium chloride hydrate.

\section{INTRODUÇÃO}

O estudo de diagramas de fases e principalmente das temperaturas eutéticas de misturas é fundamental em vários campos da química e áreas afins.

Em um diagrama de fases de uma mistura binária, é chamada de patamar eutético a temperatura onde se encontram em equilíbrio três fases: duas substâncias na fase sólida e uma solução de ambas. Por sua vez, o ponto eutético é aquele que corresponde à composição da solução na dita temperatura.

Quando uma das substâncias é a água e a outra um sal, pode ocorrer a formação de um ou mais hidratos em diferentes proporções, sal.nH $\mathrm{H}_{2} \mathrm{O}$. Alguns desses hidratos podem sofrer fusões incongruentes e, assim, gerarem patamares peritéticos. Ou seja, hidratos que se desproporcionam durante a fusão e, consequentemente, geram uma fase líquida, solução, com composição diferente daquela do hidrato.

Apesar de ser amplamente conhecido, o hidrato do cloreto de sódio, $\mathrm{NaCl} .2 \mathrm{H}_{2} \mathrm{O}$, e a fusão incongruente deste não são quase ou absolutamente mencionados nos capítulos que tratam de diagramas de fases de excelentes livros voltados para o ensino de química no terceiro grau. ${ }^{1,2}$ Isto se deve, em parte, à difícil e nem sempre inequívoca identificação dos pontos de inflexão nas curvas de resfriamento (Figura 1), particularmente, quando se parte de sistemas contendo duas fases (solução e cloreto de sódio sólido) à temperatura ambiente ou pouco acima do $0{ }^{\circ} \mathrm{C}$.

Como reforçado por Dubois, ${ }^{3}$ o sistema $\mathrm{NaCl}-\mathrm{H}_{2} \mathrm{O}$ é complicado justamente pela formação do hidrato, $\mathrm{NaCl} .2 \mathrm{H}_{2} \mathrm{O}$, que é favorecida pelo pequeno raio do cátion sódio. Entretanto, apesar das dificuldades técnicas e inerentes ao sistema, vários autores obtiveram, utilizando técnicas de resfriamento, o diagrama de fases completo do sistema $\mathrm{NaCl}-\mathrm{H}_{2} \mathrm{O}$, caracterizando em torno de $0,1^{\circ} \mathrm{C}$ o patamar relativo à fusão incongruente do hidrato. ${ }^{4-6}$

Neste trabalho, empregamos um método simples envolvendo curvas de aquecimento que se provou confiável na determinação das mudanças de fase ocorrendo por fusão e não por solidificação, como é feito normalmente.

*e-mail: lucilia_ramos@hotmail.com

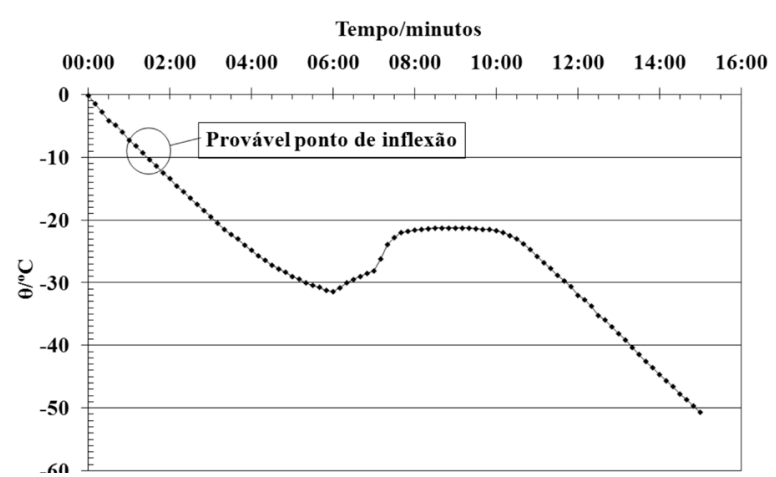

Figura 1. Curva de resfriamento do sistema $\mathrm{NaCl}-\mathrm{H}_{2} \mathrm{O} 25 \% \mathrm{p} / \mathrm{p}$ exemplificando a dificuldade de identificação dos pontos de inflexão e o significativo super-resfriamento imediatamente anterior à observação do patamar eutético

A motivação do trabalho não foi, apenas, a da construção do diagrama por si, mas, também, a de esboçar e desenvolver um método simples que pudesse ser utilizado, com as devidas modificações, na caracterização, ou na emulação de mudanças de fases que ocorram durante os estudos microtermométricos de Inclusões Fluidas (IF), porções microscópicas de fluidos aprisionados nos minerais. . $^{4,5,7,8}$ Por exemplo, estudos de inclusões fluidas presentes em minerais associados à paragênese uranífera de Lagoa Real, BA, resultaram na confirmação da existência do íon sódio nesses fluidos que, no caso, possuem baixas temperaturas iniciais de fusão (entre -70 e -50 $\left.{ }^{\circ} \mathrm{C}\right){ }^{7,9}$ Assim sendo, seria importante e esclarecedor se determinar, o mais exatamente possível, a composição destes fluidos. Porém, devido aos tamanhos micrométricos dessas inclusões fluidas e ao baixo ponto de fusão obtido para os fluidos contidos nas mesmas, a determinação direta dessa composição, baseada somente nas análises microtermométricas, torna-se extremamente complexa. ${ }^{7}$ Portanto, uma abordagem alternativa poderia ser factível e, no caso, partimos para o desenvolvimento de um método que permitisse a determinação inequívoca de pontos de fusão, em torno de $50{ }^{\circ} \mathrm{C}$ negativos, de sistemas compostos. Nesse estágio do trabalho e nessa 
faixa de temperatura, observamos que a determinação da fusão era extremamente insatisfatória e pouco precisa, quando feita a partir da inflexão em curvas de resfriamento, particularmente devido aos processos associados à precipitação dos sais. Assim sendo, partimos para a abordagem oposta: determinação da fusão a partir da inflexão nas curvas de aquecimento, que se revelou exata e precisa para o sistema modelo básico, soluções de cloreto de sódio. Essa abordagem permitiu, também, ou no mínimo, a determinação inequívoca do "elusivo" patamar peritético atribuído à formação/decomposição do hidrato do cloreto de sódio.

\section{PARTE EXPERIMENTAL}

A etapa inicial consistiu na adaptação do método de resfriamento tradicional. Esta adaptação se fez necessária para que fosse possível alcançar baixas temperaturas durante o resfriamento, ao mesmo tempo em que se contrabalanceava a velocidade do mesmo.

Foram testados como fontes de resfriamento tanto o nitrogênio líquido $\left(\mathrm{PE}=-196^{\circ} \mathrm{C}\right)$ quanto o sistema gelo-seco/acetona.

Um termômetro Modelo HD2328.0 Dellt e um Termopar do tipo $\mathrm{K}$ com capacidade de leitura em uma faixa de +1370 a $-200{ }^{\circ} \mathrm{C}$ foram utilizados durante as análises. Claro está que, no caso de se utilizar o sistema gelo seco-acetona, pôde-se utilizar um termômetro cujo limite operacional inferior fosse menor ou igual a $-80^{\circ} \mathrm{C}$.

A intensidade do fluxo de calor foi diminuída pela construção de uma fronteira de baixa permissibilidade térmica. Essa baixa permissibilidade foi obtida empregando-se um tubo falon, de polietileno, de diâmetro de aproximadamente $1,6 \mathrm{~cm}$ contendo a amostra a ser resfriada, dentro de um tubo de ensaio de vidro de diâmetro de aproximadamente $3,7 \mathrm{~cm}$, intercalado com um isolante.

Como isolante foram testados vários materiais, entre eles papel absorvente, isopor, serragem, pó de madeira, flanela de algodão e plástico bolha, na observação da fusão de padrões [água destilada $\left(\mathrm{PF}=0{ }^{\circ} \mathrm{C}\right)$, a acetona $\left(\mathrm{PF}=-95^{\circ} \mathrm{C}\right)$ e acetonitrila $\left.\left(\mathrm{PF}=-46^{\circ} \mathrm{C}\right)\right] .{ }^{10}$

Partiu-se, então, para a determinação do ponto de solidificação de misturas $\mathrm{NaCl}-\mathrm{H}_{2} \mathrm{O}$. Esta metodologia de determinação não foi, como dito, eficiente. Optou-se, portanto, por se fazer a análise térmica a partir do aquecimento dos sistemas previamente resfriados. $\mathrm{Ou}$ seja, construir o diagrama de fases a partir dos pontos de inflexão e patamares observados durante um lento e homogêneo aquecimento dos sistemas contendo apenas fases sólidas.

Inicialmente o cerne da montagem (tubo falon contendo uma mistura, ou o padrão e o termopar) era imerso no banho congelante. Após a completa solidificação do sistema, o tubo era inserido no invólucro de baixa permissividade térmica e, em seguida, exposto ao ambiente, registrando-se a temperatura a cada $10 \mathrm{~s}$.

O procedimento acima foi repetido várias vezes para a mesma mistura e, a seguir, a análise prosseguia para outros sistemas (misturas com diferentes proporções entre as fases sólidas). Mais especificamente, diferentes quantidades de cloreto de sódio eram adicionadas às soluções saturadas do sal à temperatura ambiente e, em seguida, o sistema era totalmente congelado.

\section{RESULTADOS}

Durante os testes feitos utilizando tanto o nitrogênio líquido quanto o sistema gelo-seco/acetona ambos apresentaram bons resultados. Entretanto, escolhemos o nitrogênio líquido para a realização dos experimentos por não deixar resíduos, ser relativamente mais fácil de manusear e, principalmente, pelo seu custo devido à disponibilidade do mesmo no DQ-UFMG. Lembrando, também, que acetona é uma substância cuja venda e utilização é controlada pela Polícia Federal e, portanto, de uso restrito.
O rápido fluxo de calor, devido à diferença entre as temperaturas do banho congelante e a ambiente, tornaria impossível a determinação dos pontos de inflexão e mesmo dos patamares nas curvas de resfriamento. Para tanto, foi montada uma fronteira de baixa permissibilidade térmica na qual o material que apresentou maior eficiência e reprodutibilidade, quando colocado entre o tubo falon e o tubo de vidro, foi o pó de madeira que, logo, foi o escolhido e empregado nas análises subsequentes (Figura 2).

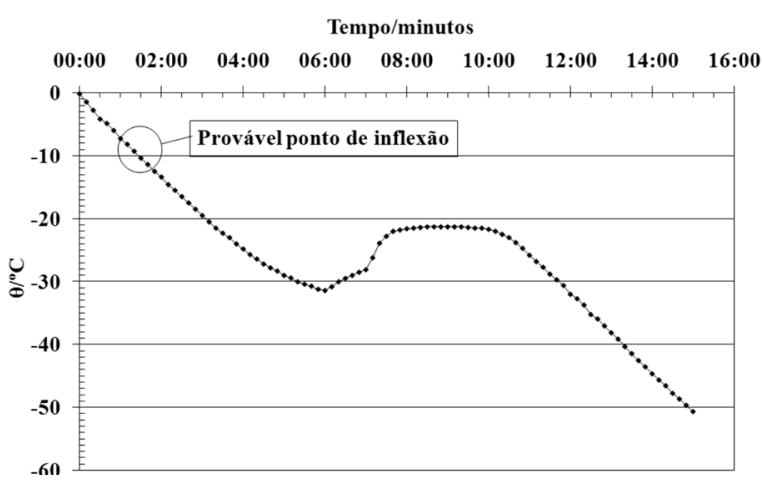

Figura 2. Curva de resfriamento da acetona utilizando como meio isolante pó de madeira

Quando se partiu para a determinação do ponto de solidificação de misturas $\mathrm{NaCl}-\mathrm{H}_{2} \mathrm{O}$ confirmou-se a impossibilidade de uma caracterização inequívoca dos pontos de inflexão e mesmo dos patamares teoricamente presentes, quando se partia de sistemas contendo duas fases ou soluções concentradas. Foi quando se optou por fazer as análises por aquecimento das amostras como descrito anteriormente.

Na Figura 3 encontram-se algumas das curvas de aquecimento obtidas, para misturas de diferentes proporções $\left(\mathrm{NaCl}_{(\mathrm{s})}-\right.$ solução saturada do sal) e soluções insaturadas à temperatura ambiente.

Ao final, a análise térmica das curvas de aquecimento desses diferentes sistemas permitiu a construção do diagrama de fases do sistema $\mathrm{NaCl}-\mathrm{H}_{2} \mathrm{O}$ com a devida caracterização do patamar peritético (Figura 4).

\section{CONCLUSÃO}

Apesar de acetona e acetonitrila não apresentarem um perfeito patamar de fusão, devido à presença de impurezas, elas podem ser utilizadas, dentro de uma incerteza, na calibração do termômetro e para o teste da intensidade do fluxo do calor através da fronteira (permissibilidade térmica da fronteira).

Obteve-se, também, sucesso no desenvolvimento de uma fronteira de baixa permissibilidade térmica e, para todos os efeitos, o emprego de pó de madeira (serragem fina) se mostrou superior aos demais isolantes testados, tanto na análise por resfriamento quanto na análise por aquecimento. $\mathrm{O}$ emprego do método de resfriamento, mesmo com a fronteira acima descrita, continua limitado aos sistemas contendo apenas soluções diluídas à temperatura ambiente.

Por sua vez, o método de aquecimento aqui descrito pode ser empregado para soluções em quaisquer concentrações e sistemas contendo uma fase líquida e uma fase sólida à temperatura ambiente, porque o aquecimento lento aparentemente elimina os erros associados à precipitação (mudança de solubilidade com a temperatura) do sal e à formação do hidrato/caracterização do patamar peritético. Particularmente neste sistema, onde a fusão do hidrato ocorre a 0,1 ${ }^{\circ} \mathrm{C}$, muito próxima do ponto de fusão da água pura. 
(A)

Tempo/minutos

00:00 05:00 10:00 15:00 20:00 25:00 30:00 35:00 40:00 45:00 50:00 55:00

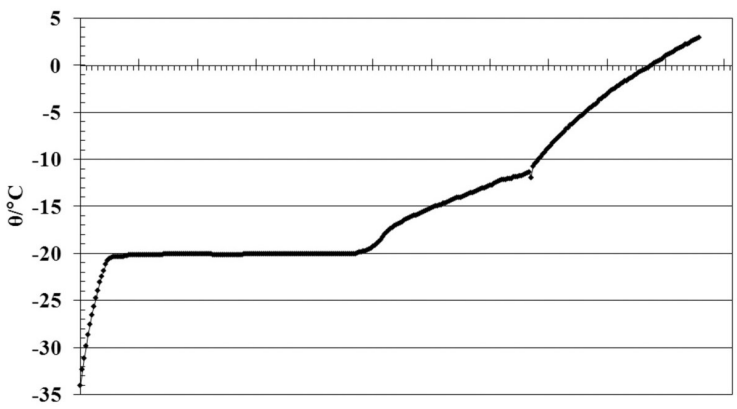

(B)

Tempo/minutos

00:00 05:00 10:00 15:00 20:00 25:00 30:00 35:00 40:00 45:00 50:00 55:00

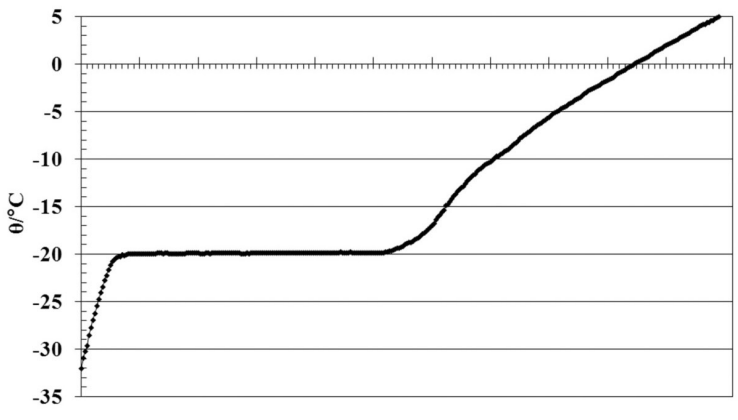

(C)

Tempo/minutos

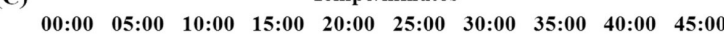
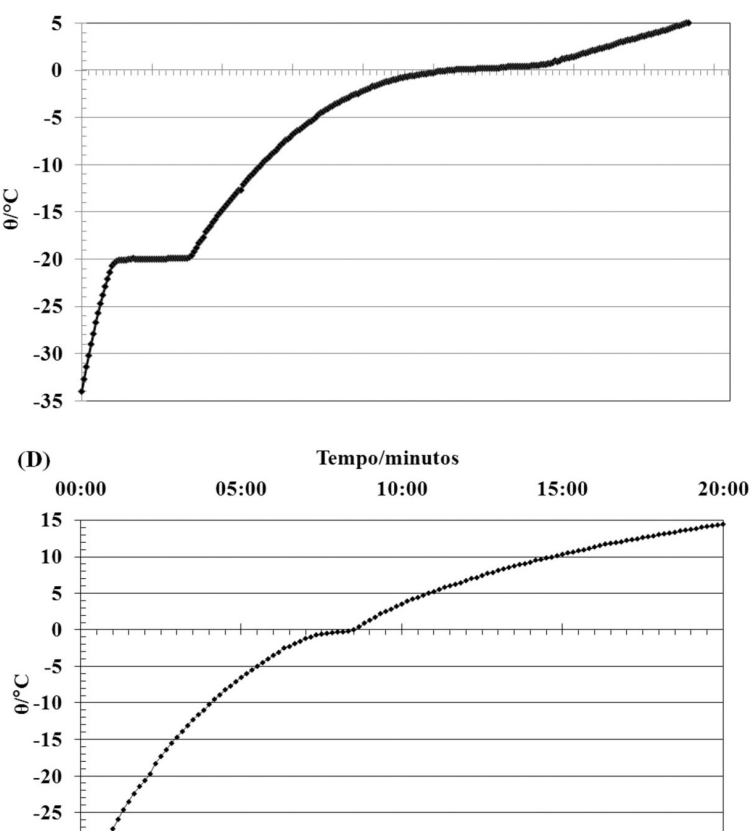

Figura 3. Curvas de aquecimento dos sistemas $\mathrm{NaCl}-\mathrm{H}_{2} \mathrm{O}$ de concentração de (A) $15 \% \mathrm{p} / \mathrm{p}$, (B) $26 \% \mathrm{p} / \mathrm{p}$, (C) $50 \% \mathrm{p} / \mathrm{p}$ e (D) $92 \% \mathrm{p} / \mathrm{p}$ de $\mathrm{NaCl}$, respectivamente

Logo, a análise térmica a partir do aquecimento lento e homogêneo, obtida via uma fronteira de baixa permissibilidade, é mais útil, menos sujeita a erros, mais fácil de ser empregada e, portanto, gera dados inequívocos, o que o tradicional método de caracterização dos pontos de inflexão em curvas de resfriamento nem sempre proporciona. Consequentemente, essa abordagem é extremante útil para

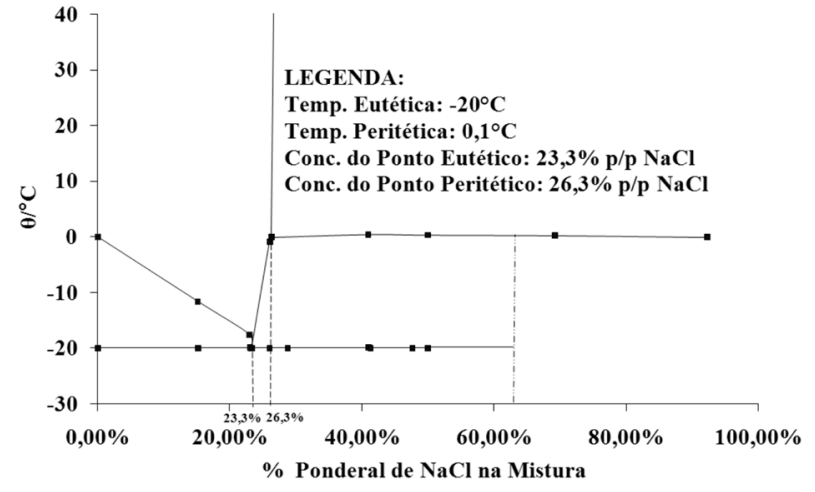

Figura 4. Diagrama de fases $\mathrm{NaCl}-\mathrm{H}_{2} \mathrm{O}, \mathrm{p}=\mathrm{cst}$

casos onde o sistema apresenta duas fases à temperatura ambiente e/ou quando o sistema é formado por soluções concentradas (perto da temperatura de saturação).

Este trabalho tem grande relevância científica e educacional. Científica por trazer um método que com devidas adaptações pode ser expandido para o desenvolvimento do diagrama de fases de diversos sistemas diferentes do supracitado, provavelmente até mesmo de sistemas com composições mais complexas do que esse. Além de ser economicamente viável, eficiente e prático para a determinação do ponto de inflexão de misturas sal/água em qualquer proporção.

A relevância educacional ocorre, pois o presente trabalho pode auxiliar os estudantes em uma melhor compreensão de sistemas que possuam o mencionado patamar peritético já que, como mencionado anteriormente, esse é pouco, ou nunca, mencionado em situações cotidianas e também em livros didáticos renomados.

\section{AGRADECIMENTOS}

À Comissão Nacional de Energia Nuclear (CNEN), ao Centro de Desenvolvimento da Tecnologia Nuclear (CDTN), à Universidade Federal de Minas Gerais (UFMG) e ao Conselho Nacional de Desenvolvimento Científico e Tecnológico (CNPq).

\section{REFERÊNCIAS}

1. Castellan, G. W.; Fundamentos de Físico-Química, $1^{\mathrm{a}}$ ed., Ao Livro Técnico: Rio de Janeiro, 1986, vol. 1, cap. 15.

2. Atkins, P.; Paula, J.; Físico-Química, $7^{\mathrm{a}}$ ed., LTC: Rio de Janeiro, 2003, vol. 1 , cap. 8 .

3. Dubois, M.; Weisbrod, A.; Shtuka, A.; Matínez-Serrano, R.; Eur. J. Mineral. 1997, 9, 987.

4. Fuzikawa, K.; Contribuições à Geologia e à Petrologia, Boletim Especial do Núcleo de Minas Gerais - SBG 1985, p. 29.

5. Roedder, E.; Econ. Geol. 1962, 57, 1045.

6. Linke, W. F.; Solubilites of inorganic and metal organic compounds, $4^{\text {th }}$ ed., Am. Chem. Soc.: New York, 1965.

7. Oliveira, L. A. R.; Rios, F. J.; Chaves, A. O.; Pereira, A. R. P.; Resumos da International Nuclear Atlantic Conference (INAC), Santos, Brasil, 2007.

8. Roedder, E.; Fluid Inclusions, Reviews in Mineralogy, Min. Soc. Amer.: Washington, 1984, vol. 12.

9. Chaves, A. O.; Tubrett, .M.; Rios, F. J.; Oliveira, L. A. R.; Alves. J. V.; Fuzikawa, K.; Correia Neves, J. M.; Matos, E. C. De; Chaves, A. M. D. V.; Prates, S. P.; Revista de Geologia. 2007, 20, 141.

10. http://www.merck.com.br/, acessada em Janeiro 2008. 